\title{
Efficient Construction of the Kedarcidin Chromophore Ansamacrolide
}

\author{
Yasuhito Koyama, Martin J. Lear, ${ }^{,}, \dagger$ Fumihiko Yoshimura, Isao Ohashi, \\ Tomoko Mashimo, and Masahiro Hirama*
}

Department of Chemistry, Graduate School of Science, Tohoku University, COE,
Sendai 980-8578, Japan

martin@ykbsc.chem.tohoku.ac.jp; hirama@ykbsc.chem.tohoku.ac.jp

Received November 4, 2004

\section{ABSTRACT}

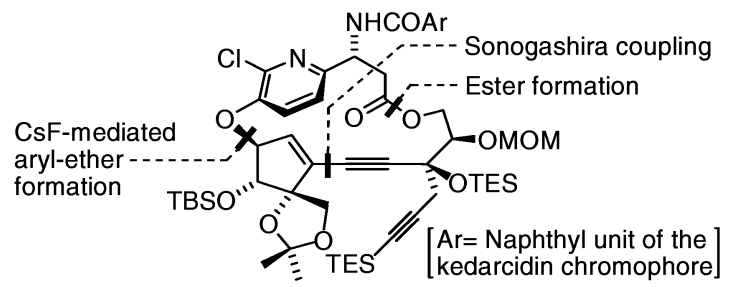

The streamlined assembly of the ansamacrolide framework of the kedarcidin chromophore via an efficient atropselective Sonogashira coupling step is described. To this end, two newly improved practical syntheses of the cyclopentene and diyne fragments have been developed, which feature $0.2 \mathrm{~mol} \%$ catalytic loadings for an $\mathrm{RCM}$ step and $i-\mathrm{PrMgCl} / \mathrm{CH}_{2} \mathrm{I}_{2}$ as a new entry to gem-disubstituted epoxides from ketones, both being applicable to $49-\mathrm{g}$ scales.

The kedarcidin chromophore (1) is a highly unstable, complex natural product that possesses, among other structural challenges, a conformationally defined ansamacrocyclic bridge. ${ }^{1}$ Although 1 has yet to be completed in its entirety, Myers et al. have constructed the whole aglycon unit of $\mathbf{1}$ through an impressive transannulation protocol. ${ }^{2}$ In our work, we have developed direct $\alpha$-selective protocols for both of the 2-deoxysugars ${ }^{3}$ and have centered on the established success of the $\mathrm{CeCl}_{3} / \mathrm{LiN}(\mathrm{TMS})_{2}$-mediated cyclization protocol to form the nine-membered, bicyclic core. ${ }^{4}$ As part of

\footnotetext{
Current address: Department of Chemistry, 3 Science Drive 3, National University of Singapore, Republic of Singapore 117543. Email: chmlmj@ nus.edu.sg.

(1) Isolation of kedarcidin and chromophore structure (1): (a) Lam, K. S.; Hesler, G. A.; Gustavson, D. R.; Crosswell, A. R.; Veitch, J. M.; Forenza S.; Tomita, K. J. Antibiot. 1991, 44, 472. Hofstead, S. J.; Matson, J. A.; Malacko, A. R.; Marquardt, H. J. Antibiot. 1992, 45, 1250. (b) Leet, J. E.; Schroeder, D. R.; Langley, D. R.; Colson K. L.; Huang, S.; Klohr, S. E.; Lee, M. S.; Golik, J.; Hofstead, S. J.; Doyle, T. W.; Matson, J. A. J. Am. Chem. Soc. 1993, 115, 8432. (c) Structure revision and synthesis of fragments 5 and 7: Kawata, S.; Ashizawa, S.; Hirama, M. J. Am. Chem. Soc. 1997, 119, 12012.

(2) Myers, A. G.; Hogan, P. C.; Hurd, A. R.; Goldberg, S. D. Angew. Chem., Int. Ed. 2002, 41, 1062.

(3) (a) L-Mycarose glycosylation study: Lear, M. J.; Yoshimura, F.; Hirama, M. Angew. Chem., Int. Ed. 2001, 40, 946. (b) L-Kedarosamine glycosylation study: Ohashi, I.; Lear, M. J.; Yoshimura, F.; Hirama, M. Org. Lett. 2004, 719.
}

our intensive efforts to provide gram quantities of late-stage targets, we describe in this letter the practical preparation of both the cyclopentene and diyne fragments and the advantage of having an appropriate C4-stereochemistry ${ }^{5}$ for the efficient assembly of the ansamacrolide framework $2{ }^{6}$

In this and our earlier work, the kedarcidin chromophore 1 was retrosynthetically stripped of its sugar components and reduced to the ansamacrolide subtargets 2 and 3 (Scheme 1). Among several macrolide-forming methods tested, the intramolecular Sonogashira coupling was the only method found powerful enough to successfully prepare ansamacrolides such as $\mathbf{3}$. In the earlier cases tested, the $\mathrm{C} 4$-epimer of 3 was targeted and yields fell into the $40-45 \%$ range. Subsequent efforts at improving the coupling yield were

(4) Examples in advanced synthetic settings: (a) Kawata, S.; Yoshimura, F.; Irie, J.; Ehara, H.; Hirama, M. Synlett 1997, 250. (b) Kobayashi, S.; Ashizawa, S.; Takahashi, Y.; Suigura, Y.; Nagaoka, M.; Lear, M. J.; Hirama, M. J. Am. Chem. Soc. 2001, 123, 11294. (c) Das, P.; Mita, T.; Lear, M. J.; Hirama, M. Chem. Commun. 2002, 2624. (d) Inoue, M.; Kikuchi, T.; Hirama, M. Tetrahedron Lett. 2004, 45, 6439. (e) Inoue, M.; Sasaki, T.; Hatano, S.; Hirama, M. Angew. Chem., Int. Ed. 2004, 43, 6500.

(5) This structural change also arose from unforeseen stereochemical difficulties that occurred during advanced stages directed toward the synthesis of the aglycon of $\mathbf{1}$. This work will be detailed elsewhere.

(6) For our preliminary synthetic study of the C4-epimer of $\mathbf{3}$, see: Yoshimura, F.; Kawata, S.; Hirama, M. Tetrahedron Lett. 1999, 40, 8281. 
Scheme 1. Retrosynthesis of Kedarcidin Chromophore $\mathbf{1}$ to Atropisomeric Ansamacrolide Subtargets 2 and 3

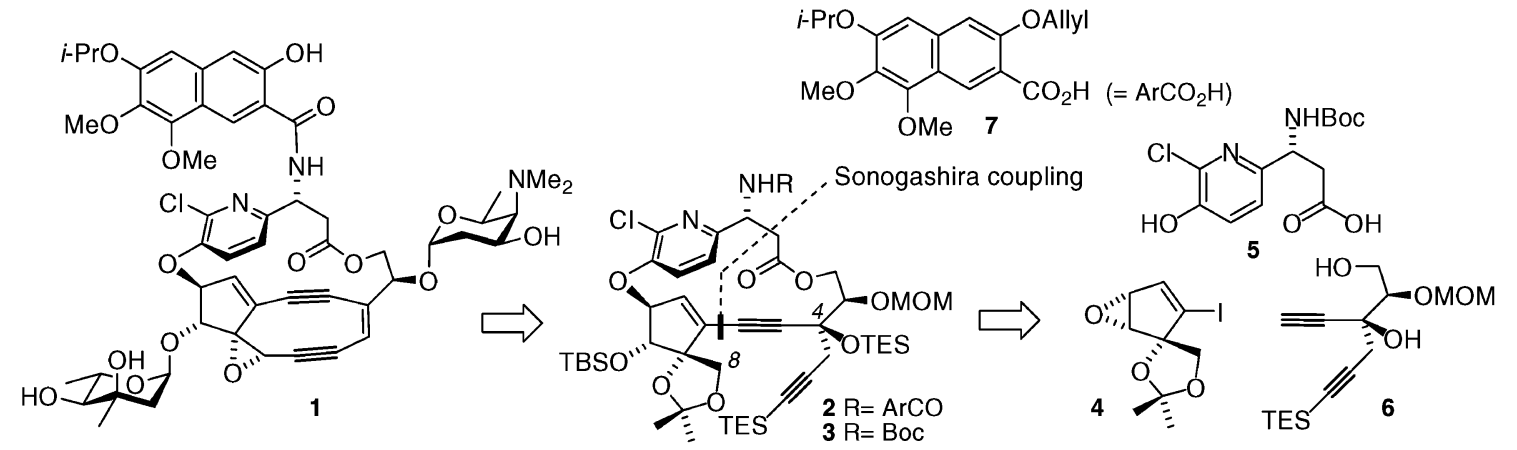

unrewarding. Eventually we surmised that with an appropriately protected precursor the targeting of the $\mathrm{C} 4$-epimer as shown (3) would reduce the ensuing transannular interactions between the chloropyridine and the projecting C8hydrogens and likely improve the coupling yield. ${ }^{5}$

Encouraged by modeling studies on $3{ }^{7}$ we set out on the development of efficient routes that would enable the largescale production of the requisite fragments 4-7 (Scheme 1). The key fragments that mostly required reassessment in their synthesis were identified as the cyclopentene (4) and diyne (6) fragments (Scheme 1). Targeting the highly oxygenated cyclopentene 4 , we began by developing a practical synthesis of the useful building block 13, which we had previously established as a good precursor to 4 (Scheme 2). ${ }^{6,8}$

Scheme 2. Practical Synthesis of Cyclopentenol 13
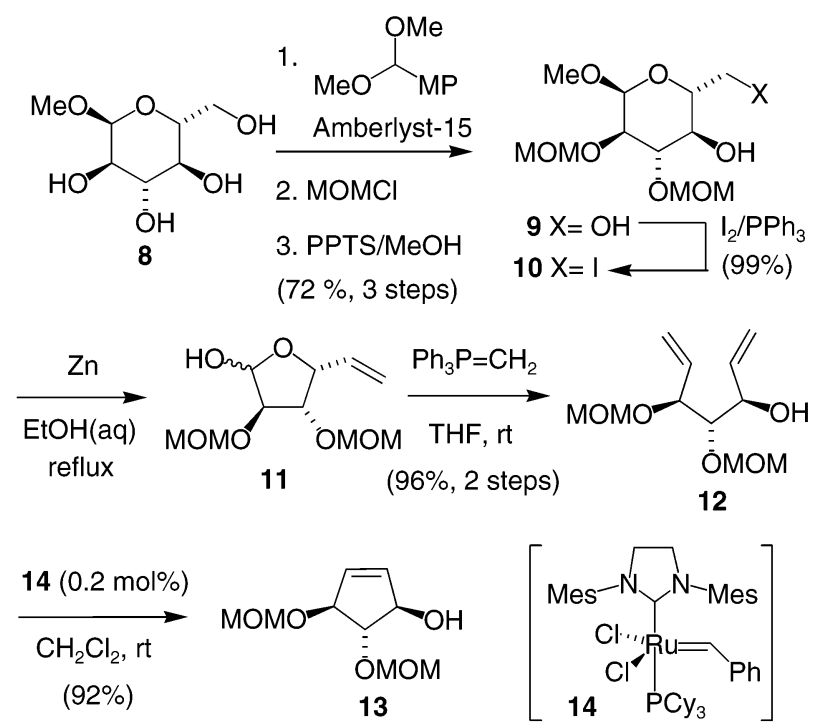

During the rising popularity of RCM, syntheses of products akin to $\mathbf{1 3}^{8}$ became simplified, and several reports detailing related routes have emerged..$^{9}$ Independent to these reports, we devised an efficient route to $\mathbf{1 3}$ from the convenient sugar $\mathbf{8}$ and highlight here the power of RCM to large-scale synthesis.
The methyl glycoside $\mathbf{8}$ was first protected as its known MPM-acetal ${ }^{10}$ and the remaining hydroxy groups were MOM-protected. Chemoselective removal of the MPM-acetal to the diol 9 and introduction of iodine at the primary position yielded the iodide 10. Reductive opening of $\mathbf{1 0}$ with zinc dust $^{11}$ under hydrolytic conditions furnished the alkenealdehyde $\mathbf{1 1}$ in its hemiacetal form, which was directly olefinated to efficiently afford the bisalkene $\mathbf{1 2}$.

Next, various RCM conditions for $\mathbf{1 2}$ were investigated. ${ }^{12}$ When Grubbs' first generation catalyst $\mathrm{Cl}_{2}\left(\mathrm{PCy}_{3}\right)_{2} \mathrm{Ru}=\mathrm{CHPh}$ was used, an enone (cf. 20, Scheme 4) was generated as a side product with 13. Although this result was desirable for the purpose of making the cyclopentene fragment $\mathbf{4}$, this reaction could not be adequately controlled on a large scale. Having performed several trials, the optimized conditions to $\mathbf{1 3}$ involved using catalytic amounts of Grubbs' second generation catalyst 14 in $0.05 \mathrm{M} \mathrm{CH}_{2} \mathrm{Cl}_{2}$ at ambient temperatures. After 3 days, purification of the reaction over activated carbon $^{13}$ gave excellent yields of the desired cyclopentenol 13.

The efficiency and practicality of this route to $\mathbf{1 3}$ is noteworthy. Each reaction involves acceptable, non-laborintensive purification and workup procedures on multigram scales. In particular, we have regularly achieved yields of 13 surpassing $90 \%$ on 50 -g scales by using only $0.2 \mathrm{~mol} \%$ of Grubbs' catalyst $\mathbf{1 4}$ in the final RCM step.

(7) By using conformational and minimization searches with MacroModel 8.5 under the MM2* force-field, $\mathbf{3}$ was estimated to display greater degrees of freedom, a lower global energy minimum, and a larger transannular cavity than the C4-macrolide-epimer, epi-3.

(8) For our previous synthesis of chiral trihydroxylated cyclopentenes akin to 13, see: Toyama, K.; Iguchi, S.; Sakazaki, H.; Oishi, T.; Hirama, M. Bull. Chem. Soc. Jpn. 2001, 74, 997.

(9) For example: (a) Ovaa, H.; Lastdrager, B.; Codee, J. D. C.; van der Marel, G. A.; Overkleeft, H. S.; van Boom, J. H. J. Chem. Soc., Perkin Trans. 1 2002, 2370. (b) Ramana, G. V.; Rao, B. V. Tetrahedron Lett. 2003, 44, 5103. (c) Moon, H. R.; Choi, W. J.; Kim, H. O.; Jeong, L. S. Chem. Lett. 2004, 506. (d) Nayek, A.; Bauerjee, S.; Sinha, S.; Ghosh, S. Tetrahedron Lett. 2004, 45, 6457.

(10) (a) Johansson, R.; Samuelsson, B. J. Chem. Soc., Perkin Trans. 1 1984, 2371. (b) Chen, J.; Dormán, G.; Prestwich, G. D. J. Org. Chem. 1996 $61,393$.

(11) Ferrier, R. J.; Schmidt, P.; Tyler, P. C. J. Chem. Soc., Perkin Trans. $1 \mathbf{1 9 8 5}, 301$.

(12) Selected reviews on metathesis: (a) Schrock, R. R. Tetrahedron 1999, 55, 8141. (b) Furstner, A. Angew. Chem., Int. Ed. 2000, 39, 3012. (c) Grubbs, R. H. Tetrahedron 2004, 60, 7117.

(13) Cho, J. H.; Kim, B. M. Org. Lett. 2003, 5, 531. 
To establish a practical, stereoselective synthesis of the diyne fragment $\mathbf{6}$, we employed some of our previous chemistry. In short, the enediyne-diol 16 was reliably prepared in $40-45 \%$ yield in four steps from the readily available glyceraldehyde derivative 15 (Scheme 3). ${ }^{14}$ The enantiomerically pure enediyne diol 16 was subjected to Sharpless asymmetric epoxidation, (-)-SAE, using diethyl $\mathrm{D}-(-)$-tartrate at $-20{ }^{\circ} \mathrm{C}$. This furnished the $\beta$-epoxide $\mathbf{1 7}$ in $92 \%$ yield over two steps, after selective MMTr protection of the primary alcohol. Subsequent MOM protection of the secondary alcohol gave 18. The key step, the regio- and stereoselective reductive ring opening of the $\beta$-epoxide $\mathbf{1 8}$, was found most effective using our first reagent of choice, namely, Superhydride $\left(\mathrm{LiHBEt}_{3}\right)$, which under high dilution conditions in $\mathrm{Et}_{2} \mathrm{O}$ at low temperature yielded the tertiary alcohol 19 with only traces of allene side products. ${ }^{15}$ Deprotection of the MMTr group then afforded the desired diyne fragment 6 in $88 \%$ overall yield.

Having synthesized sufficient quantities of the requisite

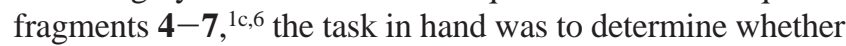
our revised plan would result in a more efficient assembly of the ansamacrolide 3 (Scheme 4). Following TPAPoxidization $^{16}$ of $\mathbf{1 3}$ to its enone and introduction of iodine to give the iodo-enone $\mathbf{2 0}$, we needed to produce the $\beta$-epoxide $\mathbf{2 1}$ on a gram scale; however, this could not be achieved reliably under the classic conditions of using the sulfonium ylid of $\mathrm{Me}_{3} \mathrm{SI}^{6,17}$ After several less productive routes and reagents were surveyed, we discovered that the simple generation (under the techniques developed by Knochel's group) and addition of $\alpha$-iodomethylmagnesium halide to the enone $\mathbf{2 0}$ yielded the $\beta$-epoxide $\mathbf{2 1}$ as a single isomer in good yields. ${ }^{18}$ Importantly, this procedure could be reliably performed starting with gram quantities of 20 (up to 49-g scales have been tested). To the best of our knowledge, the combination of $\mathrm{CH}_{2} \mathrm{I}_{2}$ and $i$-PrMgCl is new and represents a convenient entry for the generation of gemdisubstituted epoxides. ${ }^{18 \mathrm{~b}}$

Exposure of $\mathbf{2 1}$ to aqueous acid afforded the diol 22, which was protected as its cyclic carbonate, and the MOM groups were replaced with TBS ethers to furnish 23. Further successive dual exchanges of diol protective groups and epoxide formation under Mitsunobu conditions gave 4. This sequence was found far more productive than that previously established ${ }^{6}$ and importantly avoided migration of protecting groups. Coupling of $\mathbf{4}$ with the $\beta$-2-chloroazatyrosine deriva-

(14) Wang, G. X.; Iguchi, S.; Hirama, M. J. Org. Chem. 2001, 66, 2146.

(15) (a) For the classical use of $\mathrm{LiHBEt}_{3}$ to open epoxides, see: Krishnamurthy, S.; Schubert, R. M.; Brown, H. C. J. Am. Chem. Soc. 1973 95, 6. (b) In THF, moderate yields of 19 were obtained $(70-75 \%)$ accompanied by $15-20 \%$ of allene stereoisomers due to competing conjugate additions. $\mathrm{Et}_{2} \mathrm{O}$ was found to be the optimal solvent.

(16) Ley, S. V.; Norman, J.; Griffith, W. P.; Masden, S. P. Synthesis 1994, 639.

(17) Corey, E. J.; Chaykovsky, M. J. J. Am. Chem. Soc. 1965, 87, 1353.

(18) (a) For recent progress of using $i$ - $\mathrm{PrMgCl}$ to generate Grignard reagents via iodine-magnesium exchange, see: Sapountzis, I.; Lin, W.; Fischer, M.; Knochel, P. Angew. Chem., Int. Ed. 2004, 43, 4364. (b) For a related report of using $\mathrm{RCHI}_{2} / i$-PrMgCl to form 1,2-disubstituted epoxides from aldehydes, see: Schulze, V.; Nell, P. G.; Burton, A.; Hoffmann, R. W. J. Org. Chem. 2003, 68, 4546. (c) The stereochemical result $(\alpha$-facial attack) is similar to that found when sufonium ylids were used and is presumably dominated by the Burgi-Dunitz trajectory being more hindered by the C11-MOM ether on the $\beta$-face.

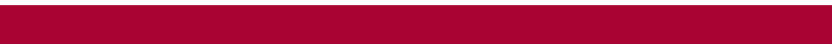

Scheme 3. Practical Synthesis of Diyne Diol 6
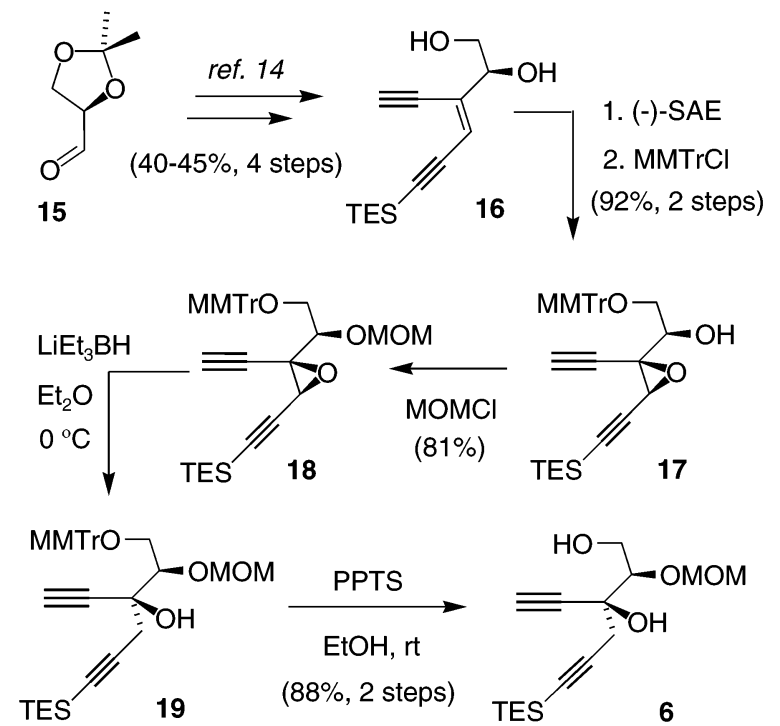

tive $5^{1 \mathrm{c}}$ according to the previously reported conditions $(\mathrm{CsF}$, DMF, $\left.60{ }^{\circ} \mathrm{C}\right)^{19}$ and subsequent TBS protection of the secondary alcohol gave the aryl ether adduct $\mathbf{2 4}$ in $90 \%$ yield over two steps.

Saponification of the methyl ester gave the carboxylic acid of 24, which was condensed selectively with the primary alcohol of the diyne $\mathbf{6}$ using EDC $\cdot \mathrm{HCl}$ and DMAP. Subsequent silylation of the tertiary $\mathrm{C} 4-\mathrm{OH}$ with $\mathrm{TESCl}$ then gave the cyclization precursor 25. Although a whole spectrum of palladium-coupling conditions were screened, the intramolecular Sonogashira coupling of $\mathbf{2 5}$ according to the previously established conditions, $\mathrm{Pd}_{2}(\mathrm{dba})_{3} \cdot \mathrm{CHCl}_{3}$ ( 0.5 equiv), CuI (2 equiv), and $i$ - $\operatorname{Pr}_{2} \mathrm{NEt}$ (30 equiv) in degassed DMF (2 $\mathrm{mM}$ ) at room temperature, still provided the optimal results and gave the ansamacrolide $\mathbf{3}$ as a single atropisomer in $90 \%$ yield. This yield was far superior to that ever obtained for the same transformation to C4-epi-3 (47\% maximum) and points to reduced transannular interactions. ${ }^{6,7}$

After several trials, the selective deprotection of the Boc group of $\mathbf{3}$ ( $\mathrm{R}=$ Boc) was efficiently achieved by initial treatment with TBSOTf to give the incipient $O$-silyl carbamate $3(\mathrm{R}=\mathrm{C}(\mathrm{O}) \mathrm{OTBS})$, which was then hydrolytically collapsed on exposure to silica to give the free amine $\mathbf{3}$ ( $\mathrm{R}$ $=\mathrm{H}) \cdot{ }^{20}$ The condensation of this amine $\mathbf{3}(\mathrm{R}=\mathrm{H})$ with the naphthoic acid $7^{1 \mathrm{c}}$ using $\mathrm{EDC} \cdot \mathrm{HCl}$ and $\mathrm{HOAt}{ }^{21}$ ultimately gave the amide 2 in good overall yield.

The stereochemistry of the atropisomer 2 was unambiguously determined by NOE ${ }^{1} \mathrm{H}$ NMR experiments. In comparison to the large $\mathrm{NOE}$ values measured between $\mathrm{H}^{\prime}$ and H10 in C4-epi-2, smaller NOEs occurred in $\mathbf{2}$ in the $0.2-0.3 \%$ range. ${ }^{6,22}$ This result again implies a larger transannular cavity for $\mathbf{2}$ than the more enclosed cavity of

(19) Kawata, S.; Hirama, M. Tetrahedron Lett. 1998, 39, 8707.

(20) Sakaitani, M.; Ohfune, Y. J. Org. Chem. 1990, 55, 870.

(21) Because of the decomposition of $\mathbf{3}(\mathrm{R}=\mathrm{H})$, HOAt was found superior to 1-hydroxybenzotriazole (HOBt): Carpino, L. A. J. Am. Chem. Soc. 1993, 115, 4397. 
Scheme 4. Assembly of Ansamacrolide 2
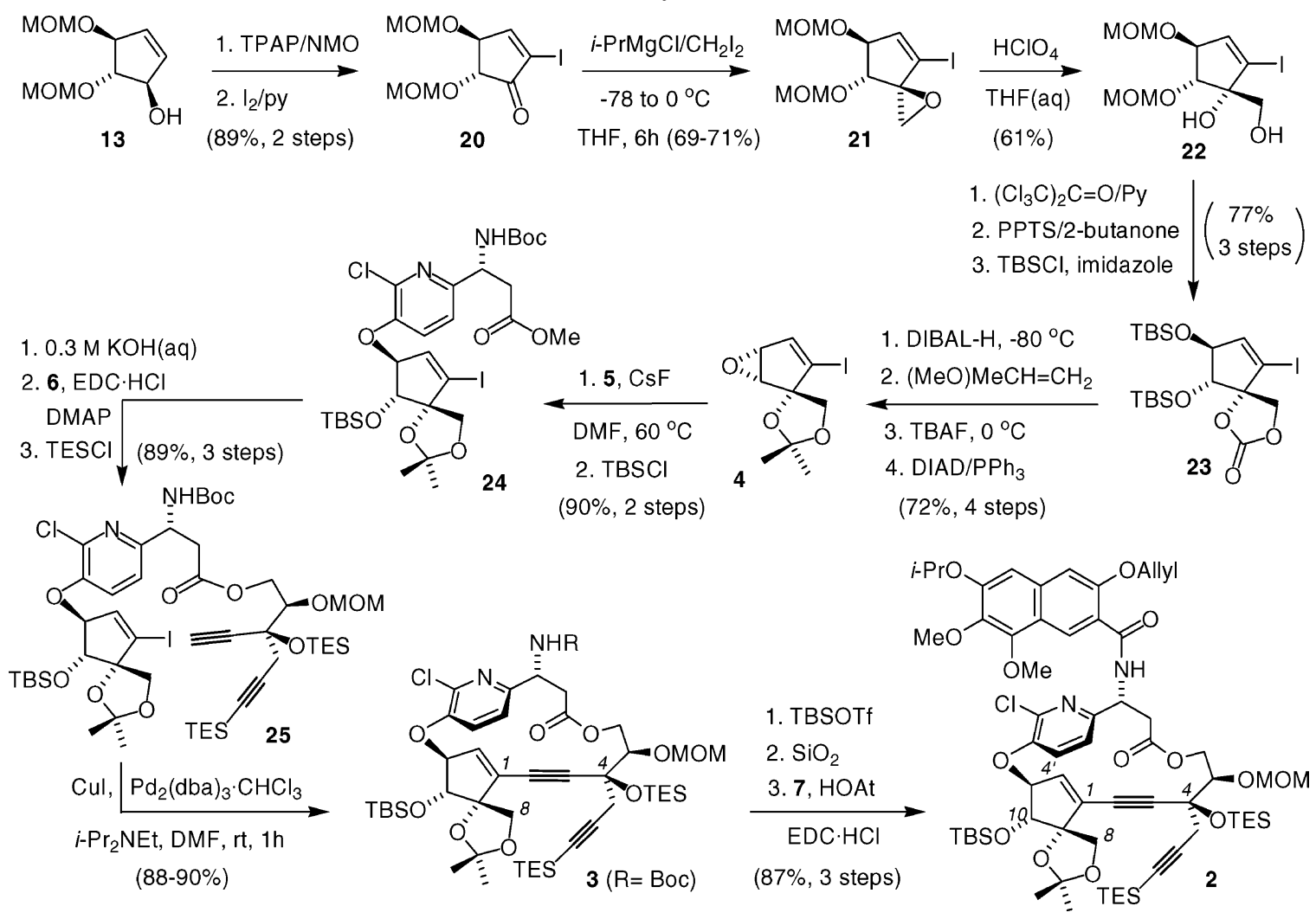

its C4-epimer. ${ }^{7}$ In addition, variable-temperature NMR studies were performed on the single atropisomers $\mathbf{2}$ and $\mathbf{3}$, which were found to be conformationally stable about the azatyrosine stereogenic axis from 25 to $100{ }^{\circ} \mathrm{C}$ (see Supporting Information). ${ }^{23}$

In conclusion, the atropselective construction of the ansamacrolide moiety of kedarcidin chromophore has been established in both an efficient and practical manner. Further studies directed toward the total synthesis of $\mathbf{1}$ are actively being pursued and will be disclosed in due course. ${ }^{5}$

(22) Similarly, between the C4-epimers of $\mathbf{3}$, only a small $(0.3 \%)$ NOE between $\mathrm{H}^{\prime}{ }^{\prime}$ and $\mathrm{H} 10$ was observed in the epimer $\mathbf{3}$, unlike the large NOEs observed for C4-epi-3 (cf. ref 6).

(23) For other observations of atropisomer behaviour, see: Myers, A. G.; Hurd, A. R.; Hogan, P. C. J. Am. Chem. Soc. 2002, 124, 4583.
Acknowledgment. This work was supported under the CREST program from JST and a Grant-in-Aid for Scientific Research, Wakate B, by MEXT (to M.J.L.). Fellowships (to F.Y. and I.O.) from the Japanese Society for the Promotion of Science for Young Japanese Scientists are gratefully acknowledged.

Supporting Information Available: Full experimental details for all new compounds and reactions are provided, including ${ }^{1} \mathrm{H}$ NMR spectra of $\mathbf{2}$ at variable temperatures. This material is available free of charge via the Internet at http://pubs.acs.org.

OL0477374 